\title{
The Role of CT Scan and Histopathology in Diagnosis of Allergic Fungal Sinusitis
}

\author{
${ }^{1}$ Bhanu Bhanu Bhardwaj, ${ }^{2}$ Jaskaran Singh Gill, ${ }^{3}$ Tanya Singh
}

\begin{abstract}
Allergic fungal sinusitis is the disease with emerging incidence in last decade. The diagnosis of AFS requires a combination of clinical, radiographic, microbiological and histopathological information. CT scan findings in AFS are considered important in preoperative diagnosis but definitive diagnosis requires histological verification. The role of CT scan as the preoperative diagnostic modality in AFS has been analyzed in 50 patients in a prospective study conducted at our institute and results have been very assuring. This article will surely satisfy many inquisitive minds about the speculations regarding the diagnosis of AFS.
\end{abstract}

Keywords: AFS, Preoperative diagnosis, Role, CT scan.

How to cite this article: Bhardwaj BB, Gill JS, Singh T. The Role of CT Scan and Histopathology in Diagnosis of Allergic Fungal Sinusitis. Clin Rhinol An Int J 2014;7(3):117-120.

Source of support: Nil

Conflict of interest: None

\section{INTRODUCTION}

Allergic fungal sinusitis (AFS) - a disease under much attention and discussion in rhinology is a noninvasive fungal disease. Precisely, it is an allergic response to the presence of extramucosal fungi within the sinuses. It has propensity for allergic mucin and mucocele formation which eventually leads to bone remodeling. ${ }^{1,2}$ It is mostly encountered in a young atopic immunocompetent adult living in warm and humid climates which is in striking contrast to the invasive fungal infections that typically affect the immunocompromised hosts. ${ }^{3,4}$

A favorable prognosis and a totally different management protocol of AFS as compared to the invasive fungal sinusitis, chronic rhinosinusitis (CRS) and eosinophillic mucin chronic rhinosinusitis (EMCRS) is what necessitates its thorough knowledge and a need for prompt

\footnotetext{
${ }^{1}$ Senior Resident (3rd Year), ${ }^{2}$ Assistant Professor

${ }^{3}$ Junior Resident (3rd Year)

${ }^{1-3}$ Department of ENT, Sri Guru Ram Das Institute of Medical Sciences and Research, Amritsar, Punjab, India
}

Corresponding Author: Bhanu Bhanu Bhardwaj, Senior Resident (3rd Year), Department of ENT, Sri Guru Ram Das Institute of Medical Sciences and Research, Amritsar, Punjab India, Phone:91-9815201845, e-mail: bhardwaj_bhanu123@ yahoo.co.in diagnosis, justifying the ongoing research world over about this disease. For the preoperative diagnosis of the disease a CT scan of nose and paranasal sinuses is one the essential pre-requisites. ${ }^{2}$

Although CT scan findings in AFS are considered important; definitive diagnosis can only be made after histopathological examination of the tissue obtained after surgical intervention. Intraoperatively as well as histopathologically the presence of allergic mucin and fungal debris is thought to be the sine qua non for diagnosis of AFS. ${ }^{5}$ Our study is a prospective study of 50 patients which analyses and emphasis the roles and need for both preoperative CT scan and postoperative histopathological confirmation of the specimen for successful diagnosis and management of AFS.

\section{PATIENTS AND METHODS}

A prospective study of 50 patients was conducted in the Department of Otorhinolaryngology at Sri Guru Ram Das Institute of Medical Sciences and Research, Amritsar, Punjab, India. The cases included in the study were selected from the patients attending our outpatient department with complaints suggestive of chronic rhinosinusitis. All the patients who fulfilled the major and minor criteria for chronic rhinosinusitis as defined by Lanza and Kennedy underwent CT scan of nose and paranasal sinuses. ${ }^{6} 3$ to $5 \mathrm{~mm}$ sections in both coronal and axial plains. Immunocompromised patients with history/evidence of diabetes mellitus and those on immunocompromising drugs (long-term antibiotics, steroids and immunosuppressive drugs) were excluded from the study.

CT scans were evaluated for the findings characteristic of allergic fungal sinusitis consisting mainly of complete unilateral or bilateral opacification of multiple sinuses with central hyperattenuation, sinus mucocele formation and pressure erosion of lamina papyracea or skull base. The central areas of hyperattenuation represent proteinaceous allergic mucin. ${ }^{2,5}$ Intrasinus hyper attenuation is relatively characteristic of AFS on CT scan and was taken as a selection criteria for the study. Out all the patients subjected to CT scan only those 50 were included who were diagnosed as allergic fungal sinusitis on preoperative CT scan. 
These cases then underwent endoscopic sinus surgery. The intraoperative findings were studied for the evidence of AFS. The presence of allergic mucin or fungal debris grossly was taken as the evidence of fungal involvement of paranasal sinuses. All surgically excised sinus mucosa and intrasinus debris (allergic mucin and fungus) were equally divided into two halves. One-half was sent for the histopathological examination and the other half was sent to microbiological department for mycological examination. The histopathological examination was carried out to study the presence or absence of allergic mucin, eosinophillic charcot leyden crystals and noninvasive fungal hyphae. The portion of surgically exised specimen used for mycologic examination was collected in sterile normal saline. $\mathrm{KOH}$ mount $(40 \%)$ was prepared from the homogenized and nonhomogenized tissue, to dissolve the tissue debris and make fungal hyphae more clear. This $\mathrm{KOH}$ mount was then seen under microscope (40× magnification) to screen for fungal elements. The sample which was positive for fungal hyphae were further cultured. The fungal growth obtained on Sabouraud Dextrose Agar after incubation at $30^{\circ} \mathrm{C}$ and $37^{\circ} \mathrm{C}$ for 18 to 24 hours was considered as definitive.

The CT findings and operative findings were plotted on a performa with reference to each sinus individually for both sides separately. Histopathological presence of extramucosal noninvasive fungal hyphae in background of allergic mucin and eosinophillic infiltrates was regarded as the confirmatory diagnostic evidence for AFS. The data collected was analyzed to correlate the endoscopic surgery findings with preoperative CT scan findings with reference to the evidence of fungal involvement and various sinuses involved.

\section{RESULTS}

In our prospective study, the incidence of the disease was seen more in females (52\%) as compared to males $(48 \%)$.

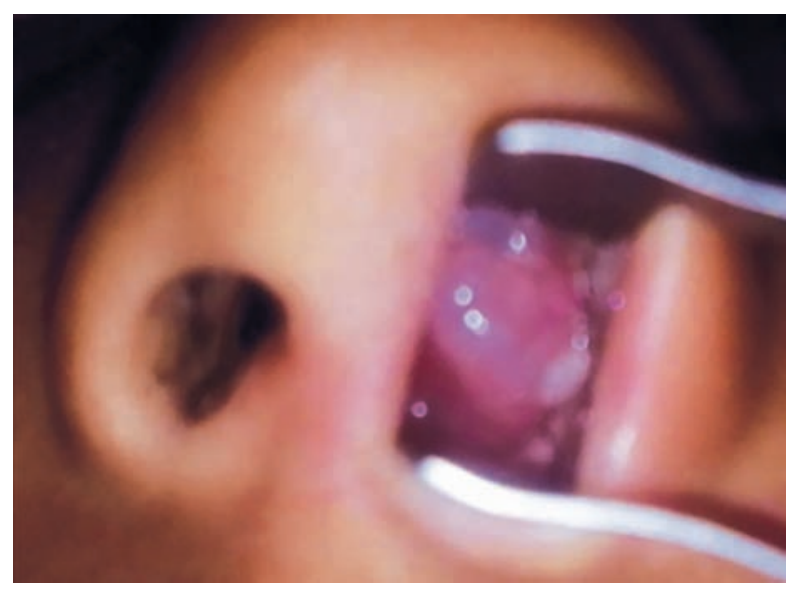

Fig. 1: Clinical photograph of AFS patient showing nasal polyposis
The band of age group predominantly affected was 20 to 30 years. The youngest patient was 8 years and the oldest was 65 years. The mean age was calculated as $26.5 \pm$ 7.61 years. Sixty-eight percent of the cases had a rural background. Nasal obstruction was present in 49 cases $(98 \%)$, nasal discharge in 46 years ( $92 \%$ ) and nasal polyposis in 43 cases (86\%) (Fig. 1). Least common symptom was telecanthus seen in only 3 cases (6\%). History of allergic rhinitis and atopy both showed a high incidence of $84 \%$ (42 cases each out of 50 cases). On preoperative CT scan, Mucosal hypertrophy and intrasinus hyperattenuation was present in $100 \%$ cases. However, remodeling of the sinus walls, bone expansion, involvement of orbit and intracranial extension was also seen in 41 cases $(82 \%), 30$ cases $(60 \%), 3$ cases $(6 \%)$ and 1 case $(2 \%)$ respectively and the disease involvement was unilateral in 27 cases and bilateral in 23 cases (Table 1) (Fig. 2). A detailed CT data of individual sinus involvement revealed the maximum incidence of involvement in maxillary sinus $(96 \%)$ closely followed by the ethmoid sinuses with an incidence of $90 \%$. Frontal sinus was the least common to be involved, with an incidence of $36 \%$. Multiple sinus involvement was seen in $88 \%$.

Surgically 42 cases showed gross presence of allergic mucin and fungal debris (Fig. 3) out of the 50 cases labelled as AFS on preoperative CT scans. The intraoperative unilateral involvement was seen in 57\% (24 out of 42 cases) and multiple sinus involvement in $83.3 \%$ (35 out of 42 cases). Intraoperatively, $95.2 \%$ showed the involvement of maxillary sinus followed by ethmoid, sphenoid and frontal in descending order of incidence.

Histopathology showed the presence of allergic mucin and fungal debris along with charcot leyden crystals (38\%) and eosinophills (84\%) without mucosal invasion in 40 out of the 50 cases which underwent surgery for AFS (Fig. 4). Out of 50 patients diagnosed as AFS on

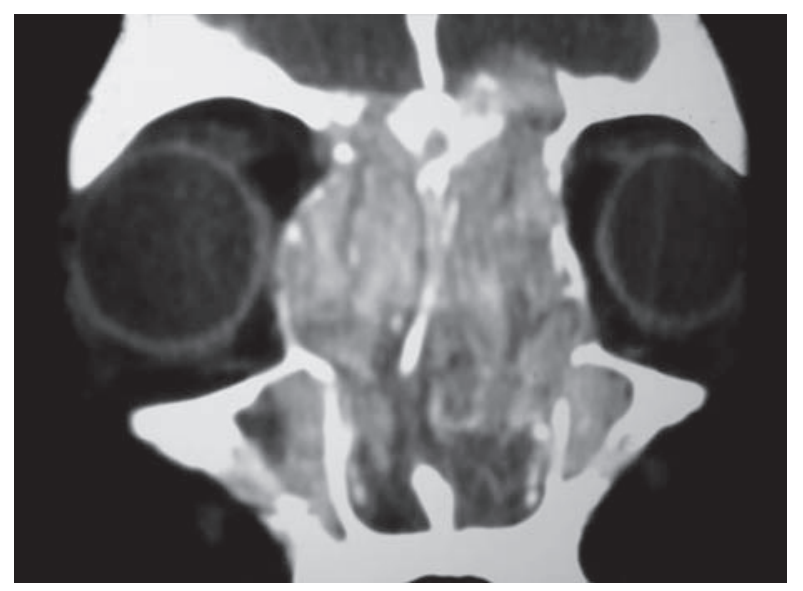

Fig. 2: Coronal section of CT scan PNS showing pressure erosion of cribriform plate and intracranial extension. Remodeling and bone expansion of lamina papyracea is also seen on the right side 


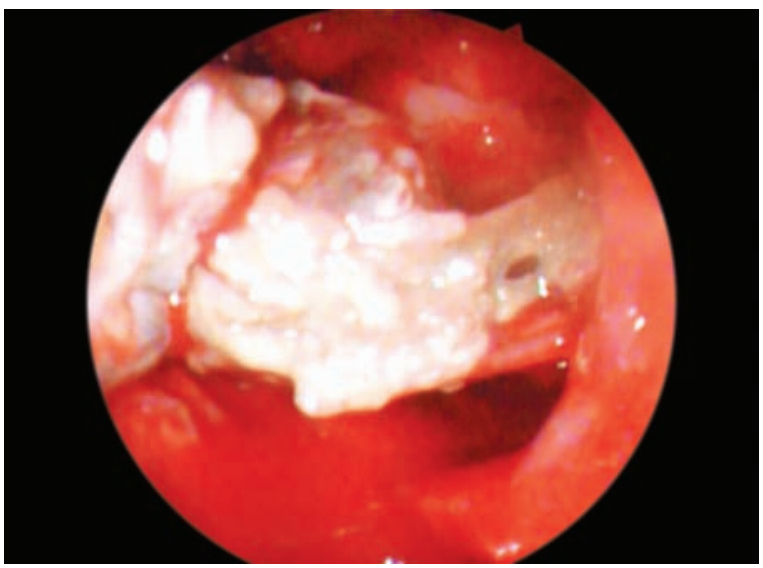

Fig. 3: View of gross fungal debris $(F)$ and allergic mucin $(A)$ in process of removal from maxillary sinus during endoscopic sinus surgery

preoperative CT findings, 42 patients showed allergic mucin grossly on surgery.

While only 40 cases demonstrated the presence of both allergic mucin and fungal hypohae in background of eosinophils and charcot leyden crystals.

\section{DISCUSSION}

Over the course of 25 years AFS has emerged as a clinically distinct form of chronic rhinosinusitis. Initially recognized for its similarities to allergic bronchopulmonary aspergillosis, now it is clear that it posses unique clinical, radiographic, pathologic and immunologic characteristics. $^{7}$ AFS is initiated when an atopic individual is exposed to inhaled fungal spores. ${ }^{8}$ The fungi deposit within a sinus cavity and an escalating immunologic reactions

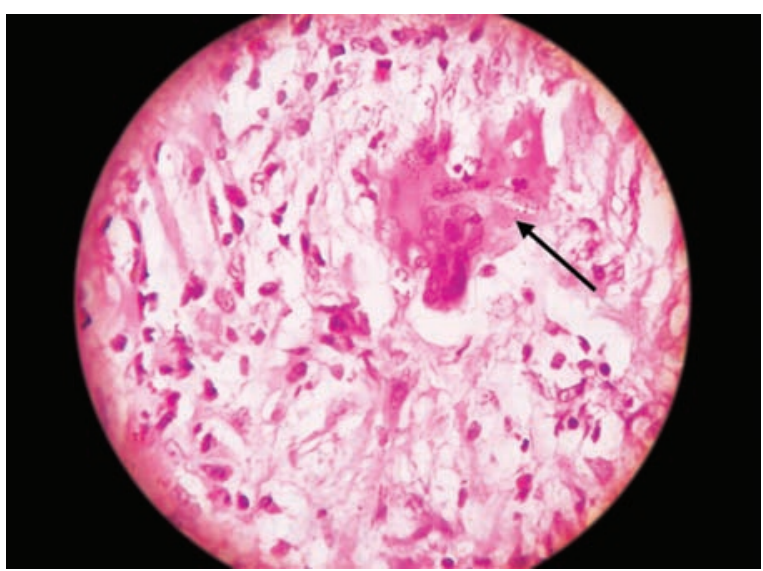

Fig. 4: 40x view showing a refractile fungal element (black arrow head) in a giant cell with no mucosal invasion typical of AFS

possibly type 1 and 3 take place against this organism causing sinonasal inflammation. The inflammation leads to polyp formation and mucus accumulation. The mucosal edema, stasis of secretions and inflammatory exudates all combine to obstruct the sinus ostia. The process then may extend to involve the adjacent sinuses and produce sinus expansion. 8,9

The diagnosis of AFS requires a combination of clinical, radiographic, microbiologic and histopathologic information. Therefore, the diagnosis of AFS cannot be made reliably after surgical intervention. ${ }^{10}$ There is still no universally recognized set of diagnostic criteria for AFS. In the absence of better defined immunologic parameters the classic and widely accepted diagnostic criteria for AFS are those described by Bent and Kuhn which include

Table 1: The comparison of CT diagnosis with operative and histopathologic diagnosis

\begin{tabular}{|c|c|c|c|c|c|c|c|c|}
\hline \multicolumn{3}{|c|}{$\begin{array}{l}\text { CT findings (in } 50 \text { selected preoperative } \\
\text { cases) }\end{array}$} & \multicolumn{3}{|c|}{$\begin{array}{l}\text { Intraoperative findings (in } 50 \text { selected } \\
\text { cases who underwent endoscopic sinus } \\
\text { surgery) }\end{array}$} & \multicolumn{3}{|c|}{$\begin{array}{l}\text { Histopathological findings } \\
\text { (of all } 50 \text { cases who underwent surgery) }\end{array}$} \\
\hline Mucosal hypertrophy & 50 & $100 \%$ & $\begin{array}{l}\text { Allergic mucin and fungal } \\
\text { debris }\end{array}$ & 38 & $78 \%$ & Allergic mucin & 40 & $80 \%$ \\
\hline Intrasinus-hyperattenuation & 50 & $100 \%$ & Fungal debris only & 04 & $08 \%$ & Noninvasive fungal hyphae & 40 & $80 \%$ \\
\hline Remodeling of sinus wall & 41 & $82 \%$ & $\begin{array}{l}\text { No fungal debris or } \\
\text { allergic mucin }\end{array}$ & 08 & $16 \%$ & Eosinophilic infiltrates & 41 & $84 \%$ \\
\hline Bone expansion & 30 & $60 \%$ & Total cases positive for & 42 & $84 \%$ & Charcot leyden crystals & 19 & $38 \%$ \\
\hline Involvement of orbit & 03 & $06 \%$ & AFS on surgery out of 50 & & & Total confirmed cases of AFS & 40 & $80 \%$ \\
\hline Intracranial involvement & 01 & $02 \%$ & preoperative cases & & & on histopathology out of 50 & & \\
\hline $\begin{array}{l}\text { Unilateral involvement of } \\
\text { diseases }\end{array}$ & 27 & $54 \%$ & $\begin{array}{l}\text { Unilateral involvement of } \\
\text { disease out of } 42 \text { cases } \\
\text { that were positive for AFS } \\
\text { on surgery }\end{array}$ & 24 & $57 \%$ & operated cases & & \\
\hline $\begin{array}{l}\text { Bilateral involvement of } \\
\text { disease }\end{array}$ & 23 & $45 \%$ & $\begin{array}{l}\text { Bilateral involvement of } \\
\text { disease out of } 42 \text { cases } \\
\text { that were positive for AFS } \\
\text { on surgery }\end{array}$ & 18 & $43 \%$ & & & \\
\hline Multiple sinus involvement & 44 & $88 \%$ & $\begin{array}{l}\text { Multiple sinus involvement } \\
\text { on surgery in } 42 \text { cases }\end{array}$ & 35 & $83.3 \%$ & & & \\
\hline
\end{tabular}

- Out of the 50 selected cases which had CT scan findings suggestive of AFS, 42 cases (84\%) demonstrated the gross findings evident of AFS intraoperatively.

- Histopathological examination of all 50 cases who underwent endoscopic sinus surgery confirmed the diagnosis in 40 cases (80\%), all these 40 cases had shown positive findings of gross allergic mucin and/or fungal debris intraoperatively. 
history of allergy, nasal polyposis, characteristic CT findings, allergic mucin without fungal invasion into sinus tissue and a positive fungal stain of sinus contents removed at surgery. ${ }^{11}$

CT scan is an important modality for the diagnosis, extension and treatment plan of the patient. CT scan of an AFS patient shows a characteristic heterogenicity of signal within the involved sinus, which has been given many names such as starry sky pattern and serpiginous pattern but commonly is referred to as double density sign, which by far describes this typical radiological appearance the best. This finding is best appreciated in soft tissue windows. ${ }^{12}$ According to Schubert, the hyperattenuating signal on CT scan in AFs may be caused by both the presence of inspissated allergic mucin and fungal hyphae within the paranasal sinuses. ${ }^{3}$

Apart from multiple opacified sinuses with central hyperattenuation, CT imaging shows sinus mucocele formation, erosion of lamina papyracea or skull base with a pushing border. ${ }^{10}$ AFS shows more bone erosion than other forms of chronic rhinosinusitis. ${ }^{13}$

Despite very sensitive CT findings, a considerable overlap exits between AFS and other forms of rhinosinusitis namely eosinophillic mucin rhinosinusitis (EMCRS,) emphasising the important role of histopathology and mycology in its diagnosis.

Grossly the allergic mucin is thick, tenacious and darkly colored. Histopathologically, this mucin consists of onion skin laminations of necrotic and degranulated eposinophills in background of fungal hyphae with occasional charcot leyden crystals. ${ }^{10}$

Though the previously accepted hallmark for the diagnosis of AFRS were the characteristic intraoperative sinus contents however, it has been noted that in some cases, the allergic mucin evacuated from sinuses does not have identifiable fungal elements. ${ }^{14}$ Ferguson described an AFS like condition with slightly different clinical features and proposed the term eosinophillic mucin chronic rhinosinusitis to describe the cases in which fungus was not identified histologically. ${ }^{15}$

The specific fungal organism has not been shown to be an important or predictive clinical characteristic, but the identification of fungus in allergic mucin either via histopathology or culture is still considered to be important to make the diagnosis of AFS. ${ }^{10}$

\section{SUMMARY AND CONCLUSION}

$\mathrm{CT}$ is one of the best diagnostic tools available for diagnosis of AFS. However, with the advent of endoscopic sinus surgery, we as otorhinolaryngologists are at an advantage as the gross presence of allergic mucin and fungal debris intraoperatively is quite evident of AFS. Moreso, with newer forms of rhinitis coming up which are phenotypically similar to AFS, the surgical material obtained should be sent for histopathological and mycological examination to confirm the presence of fungal hyphae and allergic mucin in cases of AFS.

\section{REFERENCES}

1. Schubert M. Allergic fungal sinusitis. Otolaryngol Clin North Am 2004 Apr;37(2):301-326.

2. Ryan M. Allergic fungal rhinosinusitis. Otolaryngol Clin North Am 2011 Jun;44(3):697-710.

3. Schubert MS, Goetz DW. Evaluation and treatment of allergic fungal sinusitis. I. Demographics and diagnosis. J Allergy Clin Immunol 1998 Sep;102(3):387-394.

4. McClay JE, Marple B, Kapadia L, et al. Clinical presentation of allergic fungal sinusitis in children. Laryngoscope 2002; 112(3):565-569.

5. Mukherji SK, Figueroa RE, Ginsberg LE, Zeifer BA, Marple BF, Alley JG, Cooper LL, Nemzek WR, Yousem DM, Jones $\mathrm{KR}$, et al. Allergic fungal sinusitis: CT findings. Radiology 1998 May;207(2):417-422.

6. Lanza DC, Kennedy DW. Adult rhinosinusitis defined. Otolaryngol Head Neck Surg 1997 Sep;117(3 Pt 2):S1-S7.

7. Luong A, Marple B. The role of fungi in chronic rhinosinusitis. Otolaryngol Clin North Am 2005 Dec;38(6):1203-1213.

8. Novey HS. Epidemiology of allergic bronchopulmonary aspergillosis. Immunol Allergy Clin North Am 1998;18:641-653.

9. Manning SC, Merkel M, Kriesel K, Vuitch F, Marple B. Computed tomography and magnetic resonance diagnosis of allergic fungal sinusitis. Laryngoscope 1997 Feb;107(2):170-176.

10. Ryan M. Allergic fungal rhinosinusitis. Otolaryngol Clin North Am 2011 Jun;44(3):697-710.

11. Bent JP 3rd, Kuhn FA. Diagnosis of allergic fungal sinusitis. Otolaryngol Head Neck Surg 1994 Nov;111(5):580-588.

12. Wise SK, Venkatraman G, Wise JC, DelGaudio JM. Ethnic and gender differences in bone erosion in allergic fungal sinusitis. Am J Rhinol 2004 Nov-Dec;18(6):397-404.

13. Ghegan MD, Lee FS, Schlosser RJ. Incidence of skull base and orbital erosion in allergic fungal rhinosinusitis (AFRS) and non-AFRS. Otolaryngol Head Neck Surg 2006 Apr;134(4): 592-595.

14. Allphin AL, Strauss M, Abdul-Karim FW. Allergic fungal sinusitis: problems in diagnosis and treatment. Laryngoscope 1991 Aug;101(8):815-820.

15. Ferguson BJ. Eosinophillic mucin rhinosinusitis: a distinct clinicopathologic entity. Laryngoscope 2000 May;110(5 Pt 1): 799-813. 